\title{
Diseño e Implementación de Buenas Prácticas de Manufactura (BPM) en la "Planta de Lácteos El Belén"
}

\author{
Vinicio Paredes Peralta, MsC \\ Docente de Tiempo Completo de la Facultad de Ciencias Pecuarias \\ Escuela Superior Politécnica de Chimborazo (ESPOCH), Ecuador \\ Roberto Gonzalo Socasi Simbaña, Ing. \\ Investigador, Escuela Superior Politécnica de Chimborazo \\ (ESPOCH), Ecuador \\ Fredy Erazo Rodríguez, $\mathrm{MsC}$ \\ Tatiana Sánchez Herrera, $M s C$ \\ María González Cabrera, $\mathrm{MsC}$ \\ Iván Flores Mancheno, PhD \\ Docente de Tiempo Completo de la Facultad de Ciencias Pecuarias \\ Escuela Superior Politécnica de Chimborazo (ESPOCH), Ecuador
}

Doi: 10.19044/esj.2019.v15n15p293 URL:http://dx.doi.org/10.19044/esj.2019.v15n15p293

\section{Resumen}

En la planta de lácteos "El Belén” perteneciente al cantón Quito, parroquia de Amaguaña, se elaboró y se desarrolló un manual de Buenas Prácticas de Manufactura (BPM), para lo cual se inició la investigación aplicando un Check list de diagnóstico, basado en el reglamento de Buenas Prácticas para Alimentos procesados, vigente bajo Decreto Ejecutivo 3253 estipulado por el Ministerio de Salud Publica 2002. El trabajo experimental estuvo conformado por 6 muestras de quesos frescos con 3 repeticiones cada una. Luego de aplicadas las debidas medidas correctivas en términos de calidad e inocuidad alimentaria los resultados mostraron antes de la aplicación de BPM presentaba un 54,76 \% de cumplimiento y después presentó un 81,75 $\%$ de cumplimiento de acuerdo al reglamento estipulado por parte del MSP (2002) ; en cuanto a los análisis microbiológicos de los quesos frescos, luego de la aplicación de medidas de higiene y seguridad mostraron una disminución considerable en la carga microbiológica en las poblaciones de Staphylococcus aureus con $0.25 \mathrm{UFC/g}$, mientras que para Escherichia coli y Enterobacterias su disminución fue total y en el caso de Salmonellas spp y Listeria monocytogenes hubo una ausencia total antes y después de la aplicación BPM. Resultados que permiten alcanzar una certificación BPM, para una planta 
procesadora de alimentos, puesto que debe cumplir un mínimo del $80 \%$ de los reglamentos del decreto ejecutivo 3253 del Ecuador, determinándose que la ejecución del manual de BPM en esta planta procesadora de lácteos fue efectiva.

Palabras Claves: Buenas Prácticas de Manufactura, Inocuidad alimentaria, Alimentos procesados, Carga microbiológica

\title{
Design and Implementation of Good Manufacturing Practices (GMP) at the" EI Belén Dairy Plant"
}

\author{
Vinicio Paredes Peralta, $\mathrm{MsC}$ \\ Docente de Tiempo Completo de la Facultad de Ciencias Pecuarias \\ Escuela Superior Politécnica de Chimborazo (ESPOCH), Ecuador \\ Roberto Gonzalo Socasi Simbaña, Ing. \\ Investigador, Escuela Superior Politécnica de Chimborazo \\ (ESPOCH), Ecuador \\ Fredy Erazo Rodríguez, $\mathrm{MsC}$ \\ Tatiana Sánchez Herrera, MsC \\ María González Cabrera, MsC \\ Iván Flores Mancheno, PhD
}

Docente de Tiempo Completo de la Facultad de Ciencias Pecuarias Escuela Superior Politécnica de Chimborazo (ESPOCH), Ecuador

\begin{abstract}
In "El Belén" dairy factory belonging to Quito canton, of Amaguaña parish, a manual of Good Manufacturing Practices (GMP) was made and developed, for which the research began by applying a diagnostic check list, based on in the regulation of Good Practices for Processed Food, in force under Executive Decree 3253 stipulated by the Ministry of Public Health 2002. The experimental work consisted of 6 samples of fresh cheeses with 3 repetitions each. After applying the pertinent corrective measures in terms of quality and food safety the results showed that before the application of GMP had a $54.76 \%$ compliance and then presented an $81.75 \%$ compliance according to the regulation stipulated by the MPH (2002). Regarding the microbiological analysis of fresh cheeses, after the application of hygiene and safety measures showed a considerable decrease in the microbiological load
\end{abstract}


in the populations of Staphylococcus aureus with $0.25 \mathrm{CFU} / \mathrm{g}$. While for Escherichia coli and Enterobacteiaceae their decrease was total and in the case of Salmonellas spp and Listeria monocytogenes there was a total absence before and after the GMP application. Results that allow achieving a GMP certification for a food-processing factory, since it must reach a minimum of $80 \%$ of regulations of the executive decree 3253 of Ecuador, determining that the execution of the GMP manual in this dairy processing factory was effective.

Keywords: Good manufacturing practices, Food safety, Processed foods, Microbiological load

\section{Introduction}

Según el Decreto del ARCSA-DE-067-2015-GGG de acuerdo al Reglamento de registro y control sanitario ha establecido como requisito legal la certificación de operación de las plantas procesadoras de alimentos sobre la utilización de Buenas Prácticas de Manufactura (BPM), las mismas que son útiles para el diseño y funcionamiento de los establecimientos y para el desarrollo de procesos y productos relacionados con la alimentación, son un elemento primordial para asegurar la calidad y constituyen el prerrequisito junto con los Procedimientos Operativos Estandarizados de Saneamiento (POES).

En su apartado, Ramírez, D. (2010), indica que para asegurar la calidad sanitaria de los alimentos que se comercializan, es necesaria la implementación de Buenas Prácticas de Manufactura, las mismas que tienen un enfoque preventivo en el que se garantizan las condiciones higiénicas sanitarias del entorno y las etapas de producción, para que estas no se constituyan en un vector o factor riesgo de contaminación.

Según Cáceres, (2003), las ventajas de trabajar bajo las BPM es el aumento de la productividad, un alimento limpio, confiable y seguro para el cliente, alta competitividad, mejora en la imagen de la empresa, reducción de costos, disminución de desperdicios, creación de la cultura del orden y aseo en la organización. Estos beneficios se deben principalmente a su enfoque y la aplicación en casi todas las áreas de la empresa. Las Buenas Prácticas son un sistema de control de calidad e inocuidad a través de la eliminación de riesgos de contaminación de producto.

En la actualidad, la inexistencia de manuales y procedimientos que certifiquen la aplicación de Buenas Prácticas de Manufactura (BPM) para cada una de las actividades que se realizan en las empresas de producción de lácteos, es un inconveniente tanto para la producción como para la comercialización de sus productos; como es el caso de la planta de lácteos "E1 Belén", por tal razón se hace necesaria dar a conocer la composición del 
producto, fechas de elaboración, números de lote, etc., siendo esto muy trascendental en la industria alimenticia.

El Manual de Buenas Prácticas de Manufactura (BPM) para la planta de lácteos "El Belén", contiene todas las etapas que participen en la elaboración de sus productos y de esta manera permita garantizar condiciones que aseguren la elaboración de productos inocuos, en una labor compartida entre los diferentes actores que integran la cadena de producción de los alimentos, por esta razón las diferentes actividades deben estar dirigidas a propietarios y operarios de plantas que reciben, procesan y comercializan productos lácteos, incluidos también los productores de materia prima e insumos.

Por lo tanto el propósito general de la presente investigación consiste en el diseño e implementación de Buenas Prácticas de Manufactura (BPM) en la planta de lácteos "El Belén".

\section{Metodología}

\section{Unidades experimentales}

Sé consideró como unidades experimentales a muestras de queso, a las cuales se las realizaron pruebas microbiológicas; antes y después de implementar las BPM. Estas unidades experimentales consistieron en tomar 6 muestras en el producto terminado con 3 repeticiones cada una y luego transportarlas al laboratorio de microbiología de los Alimentos de la Facultad de Ciencias Pecuarias de la Escuela Superior Politécnica de Chimborazo (FCP- ESPOCH), dentro de una caja térmica, la cualse encuentra provista de geles que permiten mantener la cadena de frio.

\section{Mediciones experimentales}

Análisis Microbiológico en los quesos se realizaron antes y después de la aplicación de BPM, de acuerdo a lo establecido por la Norma INEN y la NTE INEN -ISO 707.

- Escherichia coli (UFC/g), staphylococcus aureus (UFC/g), enterobacteriaceas (UFC/g), salmonella spp en $25 \mathrm{~g}$, listeria monocytogenes en $25 \mathrm{~g}$

\section{Análisis estadísticos y pruebas de significancia}

En la planta de lácteos "El Belén" se implementó un plan de Buenas Prácticas de Manufactura, cuyos resultados fueron sometidos a las siguientes pruebas estadísticas: Chi Cuadrado, histograma de frecuencias.

Mientras que los análisis microbiológicos en el queso fresco antes y después de la implementación del plan, fueron sometidos a los siguientes análisis estadísticos: Media, máximo y mínimo, rango, desviación estándar, coeficiente de variación, histograma de frecuencias, T Student. 
4. $\quad$ Procedimiento experimental

Este proceso investigativo se dividió en las siguientes etapas:

Fase de diagnóstico: Luego de obtener la aceptación por parte del departamento pertinente acerca del desarrollo del trabajo experimental de la presente investigación se realizó un diagnóstico visual de toda la planta (instalaciones, equipos, materiales, personal, utilizando como base un Check List para evaluar la situación actual de la planta y emitir posteriores acciones correctivas para la ejecución de las BPM.

Toma de muestras: La primera toma de muestras, se la realizó antes de la implantación del manual de buenas prácticas de manufactura, tomándose el $10 \%$ de total de la producción de lotes de queso fresco, es decir, 6 quesos los cuales se tomaron por tres semanas consecutivas, es decir 6 por semana; luego estas muestras fueron empacadas al vacío de forma aséptica, etiquetadas y posteriormente transportadas en un cooler (provisto de geles refrigerantes, que permiten mantener la temperatura de conservación de muestras de queso fresco), al laboratorio de Microbiología la ESPOCH para su siembra y posterior conteo de crecimiento microbiológico. Con esto se da cumplimento al "antes" de la aplicación de los principios y medidas de higiene establecidos en el estudio.

Fase de diseño e implementación del plan: se procedió de la siguiente manera:

1.- Antes del diseño del plan de BPM, se realizó el diagnóstico de la situación de la planta.

2.- Una vez conocida la situación de la planta, se desarrollaron los análisis microbiológicos del queso fresco.

\section{Metodología de Evaluación \\ a. Análisis microbiológico de los quesos}

Los análisis microbiológicos en los quesos se desarrollaron antes y después de haber aplicado el plan BPM en la planta como se especifican a continuación:

- Determinación de Escherichia Coli, Enterobacteriaceae y Staphylococcus aureus presentes en los quesos; estandarización de muestras para análisis; siembra metodológica; incubación; recuento microbiológico, determinación de Salmonella spp en 25 g

\section{Resultados}

Los resultados obtenidos en la presente investigación señalaron que las poblaciones microbiológicas, antes y después de la aplicación de un plan de limpieza y desinfección diseñados en el manual BPM, presentan resultados muy favorables en las muestras de queso analizadas en el laboratorio de la FCP- ESPOCH, las cuales tuvieron como objetivo llegar a tener cargas 
microbiológicas que se encuentren dentro de los rangos establecidos por la norma, como se evidencia en la tabla 1.

Tabla 1. Análisis microbiológicos aplicados a los quesos frescos producidos en la Planta de Lácteos “El Belén” antes y después de la aplicación de BPM. (Paredes, A. et al. 2017)

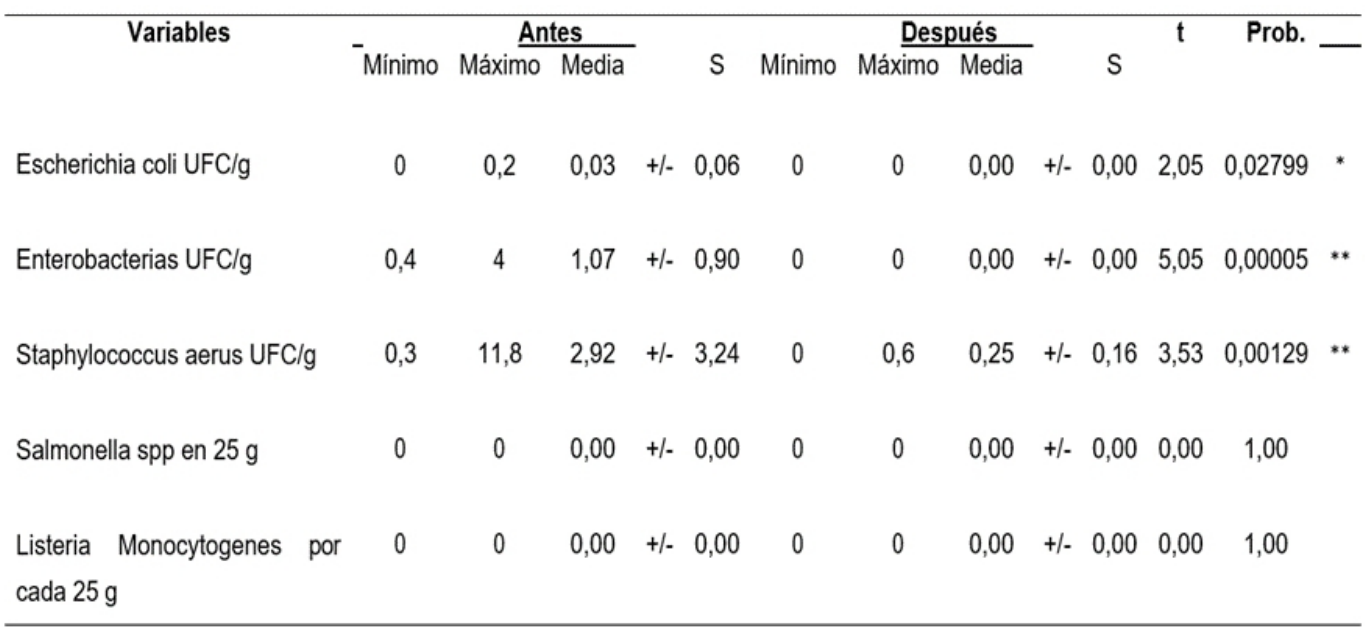

Letras iguales no difieren significativamente $(\mathrm{P}<0,05)$.

S: desviación estándar

Prob: probabilidad

\section{Escherichia coli UFC/g}

El recuento microbiológico de Escherichia coli en el queso de la planta de lácteos "El Belén" antes de la aplicación de las BPM fue de 0,03+/$0,06 \mathrm{UFC} / \mathrm{g}$, población que se redujo en su totalidad, llegando a cero, presentando diferencias significativas $(\mathrm{P}<0,05)$, poblaciones que se encuentran por debajo de la norma NTE INEN 1528: 2012 para queso fresco. Ver tabla 1 y figura 1.

En el estudio realizado por Haro, J.(2016), obtuvo concentraciones microbiológicas de $1.13 \times 104 \mathrm{UFC} / \mathrm{ml}$ en el análisis microbiológicos de quesos frescos realizado en el mercado San Alfonso de la ciudad de Riobamba, valores superiores muestran las poblaciones indicadas por Trujillo, A. (2016), en quesos frescos que se expenden en el mercado de Santa Rosa de la Ciudad de Riobamba con recuentos de 88 x $104 \mathrm{UFC/g}$, reflejando una contaminación a lo largo del proceso de elaboración presumiendo que fue ser fecal o no necesariamente debido a una mala higiene y manipulación del producto, esto posiblemente se deba a una mala prácticas de condiciones asépticas en la elaboración del producto, mal almacenamiento entre otros. 


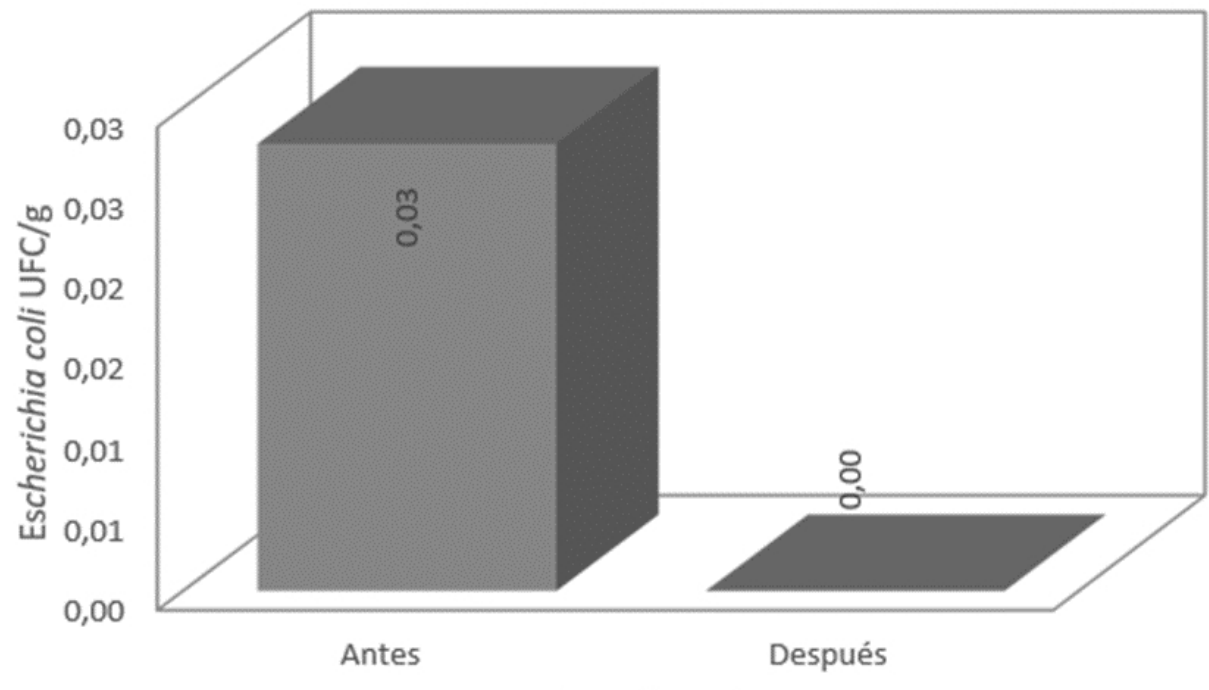

Etapa de evaluación

Figura 1. Presencia de Escherichia coli en la planta de lácteos "El Belén"

\section{Enterobacterias $U F C / g$}

En la figura 2 y tabla 1, se revelan que los recuentos de Enterobacterias en los quesos de la planta de lácteos "El Belén" antes de aplicar las BPM fue de 1,07 +/- 0,90 UFC/g, el mismo que redujo en su totalidad $(\mathrm{P}<0,01)$ es decir a cero, de esta manera se puede manifestar que la aplicación de estas normas fue eficiente cuya finalidad es garantizar la calidad de los productos en los consumidores y el mercado.

Comparando los resultados de Enterobacterias, presentadas en el estudio de Castillo, G. (2013), con concentraciones microbiológicas desde 3,66 log UFC/g hasta de 6,08 log UFC/g y los datos expresados en un artículo de la Universidad de Murcia, se puede indicar que un tratamiento inadecuado y/o contaminación posterior al tratamiento, más frecuentemente a partir de materias primas, equipos sucios o manejo no higiénico, conlleva a una multiplicación microbiana que puede permitir el crecimiento de toda la serie de microorganismos patógenos y toxigénicos, esto se demostrará al analizar los recuentos en superficies. (Grijalva, J. 2011). 


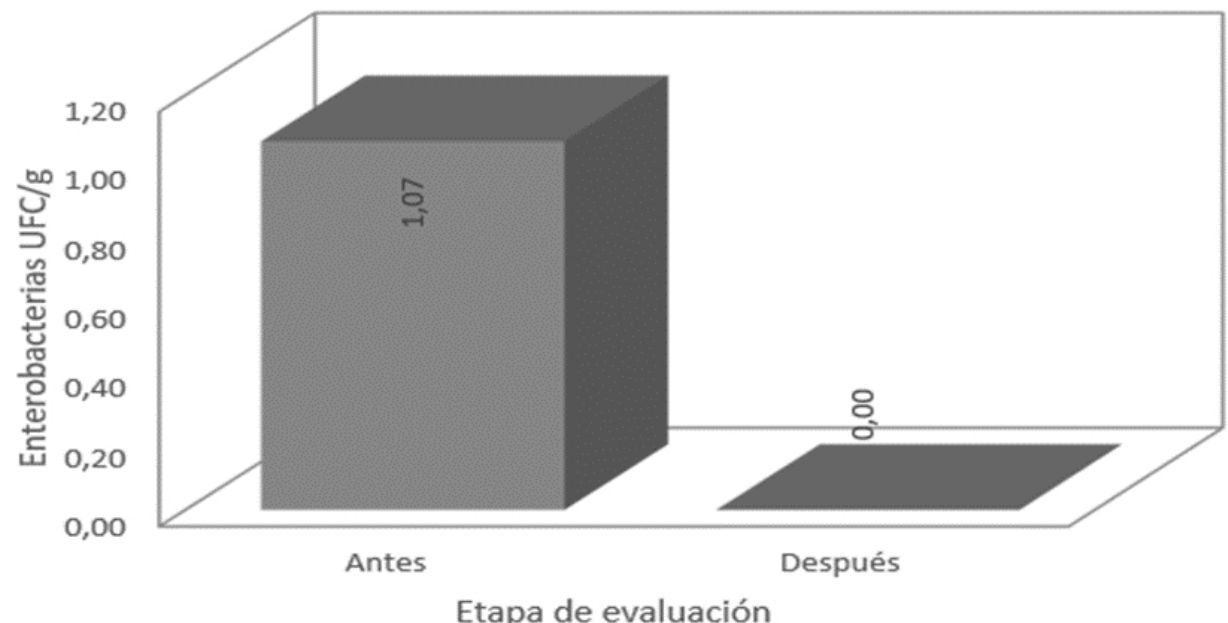

Figura 2. Presencia de enterobacterias en la planta de lácteos "El Belén"

\section{Staphylococcus aureus $U F C / g$}

El contenido de Staphylococcus aureus determinados en los quesos que se elaboran la planta de lácteos "El Belén" antes de aplicar las BPM se encontró 2,92 +/- 3,24 UFC/g de este tipo de bacterias, y luego de aplicar BPM se redujo significativamente $(\mathrm{P}<0,01)$ a $0,25+/-0,16 \mathrm{UFC} / \mathrm{g}$, conteos que se encuentran dentro de lo establecido por la norma NTE INEN 1528.

En la figura 3 y tabla 1 se exponen la carga microbiana de Staphylococcus aureus en los quesos que se elaboran en la planta de lácteos "El Belén"; valores superiores reportó Cristóbal, R y Maurtua, D. (2003), en su estudio sobre evaluación bacteriológica de quesos frescos artesanales comercializados en Lima, Perú, con valores de 2,6 x $104 \mathrm{NMP} / \mathrm{g}$, de acuerdo a la norma peruana NTP 202.087; por otro lado investigación de Trujillo, A. (2016), presenta poblaciones de Staphylococcus aureus de 24 x104 hasta los 246 x104 UFC/g, mientras que la Norma NTE INEN 1528 de 2012 establece que el índice máximo permisible para identificar nivel aceptable de calidad es de $1000 \mathrm{UFC/g}$ lo cual indica claramente que el 100\% de las muestras al sobrepasar este valor no son aptas para el consumo humano; los resultados obtenidos evidencian que los quesos comercializados en los mercados estudiados presentan condiciones higiénicas deficientes y no cumplen lo establecido en las normas y regulaciones sanitarias vigentes. 


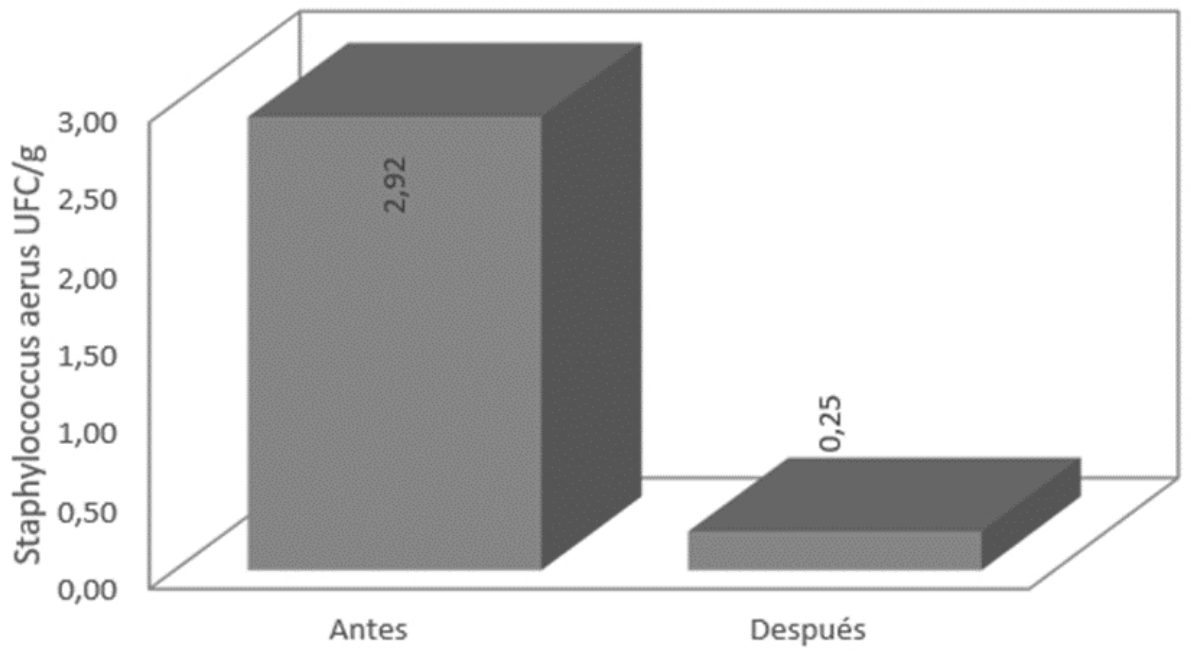

Etapa de evaluación

Figura 3. Presencia de Staphylococcus aureus en la planta de lácteos "El Belén"

\section{Salmonella spp}

En la planta de lácteos "El Belén", no se encontró Salmonella spp, en los quesos ni antes ni después de la aplicación de las BPM y POES, de esta manera se puede manifestar que siempre se ha realizado actividades de asepsia para evitar la presencia de microorganismos que causen daño en la salud de los consumidores. Ver tabla 1.

Resultados que concuerdan con los estudios realizados por Barrios, $\mathrm{H}$. (2006), acerca de la evaluación y mejoramiento de la calidad microbiológica de queso fresco, en donde no se aisló en ningún punto del proceso Salmonella spp, al igual que el estudio realizado por Quishpi, Y.( 2015), en donde al realizar el análisis microbiológico al queso para determinar presencia de Salmonella spp, el $100 \%$ de las muestras analizadas antes y después de la implementación del manual de BPM, no registraron presencia de este tipo de patógenos, determinándose entonces en estos tres estudios que el producto que se dispone es elaborado ajustándose a la legislación ecuatoriana, puesto que ellas señalan que no debe existir salmonella en los productos alimenticios, ya que son los causantes de enfermedades de tipo infecciosas al ser humano.

\section{Listeria monocytogenes por cada $25 \mathrm{~g}$}

Antes y después de aplicar las BPM en la planta de lácteos El Belén, no hubo la presencia de Listeria monocytogenes en el queso, resultados que son muy favorables para determinar la calidad e inocuidad del producto elaborado en esta planta de lácteos, señalando la eficiencia en el diseño e implementación de un manual BPM y sobre toda la acogida favorable que obtuvo, como se indica en la tabla 1. 
Contrario a los resultados obtenidos por el Instituto Nacional del Perú, en el cual de las 74 muestras de quesos estudiados se evidenciaron que 6 (21,4\%) presentaban características compatibles con L. monocytogenes, resultados similares a los obtenidos por el Ministerio de Salud y Protección Social de Colombia (2011), quienes en el 2009, en 81 muestras de queso fresco analizadas, la prevalencia de L. monocytogenes fue del $25,9 \%$ en el departamento de Boyacá estableciendo la presencia del microorganismo en la leche cruda.

2. Evaluación de porcentaje de cumplimiento de BPM en la planta de lácteos "El Belén"

A continuación en la tabla 2, se presentan los resultados de cumplimiento antes y después de la implementación de las Buenas Prácticas de Manufactura en la planta de lácteos "El Belén".

Tabla 2. Cumplimiento antes y después de las BPM en la Planta de Lácteos "El Belén" (Paredes, A. et al. 2017)

\begin{tabular}{|c|c|c|c|c|c|c|c|c|}
\hline \multirow[b]{2}{*}{ Variables } & \multirow[b]{2}{*}{ Items } & \multirow[b]{2}{*}{ Art } & \multicolumn{2}{|c|}{ Ante } & \multicolumn{2}{|c|}{ Después } & \multirow[b]{2}{*}{$\begin{array}{l}\text { Chi. } \\
\text { Cuad }\end{array}$} & \multirow[b]{2}{*}{$\begin{array}{l}\text { Prob. } \\
\text { Chi. } \\
\text { Cuad. }\end{array}$} \\
\hline & & & $\mathrm{Si}$ & No & $\mathrm{Si}$ & No & & \\
\hline Infraestructura (Instalaciones) & 44 & 13 & 23 & 21 & 37 & 7 & 33,30 & 7,9E-09 \\
\hline Equipos y Utensilios & 10 & 2 & 9 & 1 & 9 & 1 & 0,00 & 1,00 \\
\hline Personal Manipulador & 13 & 7 & 10 & 3 & 11 & 1 & 4,09 & 0,04 \\
\hline Materias primas e insumos & 6 & 6 & 2 & 4 & 4 & 2 & 3,00 & 0,08 \\
\hline Operaciones de producción & 18 & 12 & 8 & 10 & 12 & 6 & 4,00 & 0,05 \\
\hline Envasado, Etiquetado y Empacado & 10 & 8 & 8 & 2 & 9 & 1 & 1,11 & 0,29 \\
\hline Almacenamiento y Comercialización & 10 & 7 & 6 & 4 & 9 & 1 & 10,00 & $1,6 \mathrm{E}-03$ \\
\hline Control de Calidad & 8 & 5 & 3 & 5 & 7 & 1 & 18,29 & $1,9 \mathrm{E}-05$ \\
\hline Documentación & 7 & 1 & 0 & 7 & 5 & 2 & 0,00 & 1,00 \\
\hline Total & 126 & 61 & 69 & 7 & 103 & 22 & 73,79 & $2,8 \mathrm{E}-16$ \\
\hline
\end{tabular}

Prob. < 0,05: Diferencias significativas según Chi cuadrado.

\section{Infraestructura}

Antes de la implementación de las Buenas Prácticas de Manufactura, apenas se cumplían 23 actividades, las cuales mejoraron su accionar luego de la aplicación del plan BPM a un numero de 37, lo cual permitió un cambio significativo; cabe señalar que todavía no se cumplen actividades de mantenimiento, limpieza a fin de minimizar contaminaciones cruzadas; además los drenajes no cuentan con protección adecuada y no están diseñados 
de forma tal que se permita su limpieza. Ver tabla 1.

2. Equipos y Utensillos

Los equipos y utensilios de la planta, se manejaban considerando 9 actividades quedando pendiente una de ellas, de esta manera se determinó que no existió un cambio significativo ( $\mathrm{P}>0,05)$, señalándose que la actividad que necesita ser mejorada, corresponde al plan de capacitación continuo y permanente para todo el personal, en el manejo apropiado de los equipos. Ver tabla 1.

\section{Personal manipulador}

Los resultados reflejan que de las 10 actividades, 9 de ellas se desarrollan de manera eficiente, quedando pendiente una actividad que corresponde a las zonas de recepción, elaboración, envasado y almacenamiento, mismas que se encuentran en un espacio físico muy reducido y que requiere una separación, para que los procesos sean más eficientes. Ver tabla 1.

4 .

Materias primas e insumos

Las actividades que corresponden a las materias primas e insumos se han mejorado de 2 a 4, quedando pendiente una actividad que corresponde a las materias primas e ingredientes que generalmente son afectadas microorganismos. En este sentido es importante que los insumos y materias primas sean almacenadas en condiciones que impidan el deterioro, eviten la contaminación y reduzcan al mínimo su daño o alteración. Ver tabla 1.

5. Operaciones de producción

En lo relacionando a las operaciones de producción, se pudieron mejorar 4 actividades adicionales, es decir que de 8 a 12, quedando pendiente 6 actividades adicionales, determinándose que este cambio es significativo $(\mathrm{P}<$ $0,05)$, por lo tanto se puede mencionar que no se cumplen procedimientos apropiados para la elaboración de los productos, la planta no es apropiada puesto que no cuenta con áreas amplias y equipos limpios, por lo que los procedimientos de limpieza y desinfección deben ser validados periódicamente. Ver tabla 1.

6. $\quad$ Envasado, etiquetado y empacado

De las 10 actividades, 8 se cumplían antes de aplicar las BPM y solo una de ellas se logró concluir, quedando pendiente la actividad mencionada en el artículo 43 del Plan BPM, la cual trata de que en caso de que las características de los envases permitan su reutilización, será indispensable lavarlos y esterilizarlos de manera que se restablezcan las características originales, mediante una operación adecuada y correctamente inspeccionada, a fin de eliminar los envases defectuosos. Ver tabla 1.

7. Almacenamiento y comercialización

En lo relacionado al almacenamiento y comercialización de productos, de las 10 actividades destinadas a realizar, de 6 cambio a 9, 
determinándose que existe un cambio significativo $(\mathrm{P}<0,01)$, puesto que en la actualidad se cuenta con la aplicación de los artículos 52, 53, 54, 55, 57 y 59 establecidos en las BPM; no obstante, los almacenes o bodegas para almacenar los alimentos terminados deben mantenerse en condiciones higiénicas y ambientales apropiadas para evitar la descomposición o contaminación posterior de los alimentos envasados y empaquetados, también es necesaria la implementación de mecanismos para el control de temperatura y humedad que asegure la conservación de los mismos; asimismo es importante incluir un programa sanitario que contemple un plan de limpieza, higiene y un adecuado control de plagas, por último los alimentos y materias primas deben ser transportados manteniendo, cuando se requiera, las condiciones higiénico-sanitarias y de temperatura establecidas para garantizar la conservación de la calidad del producto. Ver tabla 1.

\section{Control de calidad}

Luego de la aplicación de las BPM, es imprescindible la aplicación del artículo 64, que manifiesta que la planta debe disponer de un laboratorio de pruebas y ensayos de control de calidad el cual puede ser propio o externo acreditado. Ver tabla 1.

\section{Documentación}

Se debe destacar que la planta de lácteos "El Belén", antes de la aplicación de BPM no se cumplía con ninguno de los ítems estipulados para la documentación escrita sobre instrucciones operativas, pero después de la elaboración y aplicación del manual BPM se cumplió con 5 de los 7 ítems evaluados, siendo preciso la redacción de los POES (Procedimientos Operativos Estandarizados de Saneamiento), de forma clara y precisa para que sea comprendida por el personal a cargo de su aplicación de manera más eficiente, del mismo modo adecuar un sistema de registros para cada procedimiento pre operacional, operacional y acciones correctivas a fin de disminuir errores. Ver tabla 1.

\section{Conclusiones}

El Manual de Buenas Prácticas de Manufactura es una herramienta que nos permite mejorar los aspectos de cumplimiento con la norma establecida en cuanto a las instalaciones, infraestructura, equipos y utensilios, operaciones de producción, materias primas, el transporte y comercialización; el cual fue creado tomando en cuenta las características presentadas en el check list acorde a la realidad de la empresa en estudio.

La capacitación al personal en temas de BPM, buenas prácticas de producción, tanto administrativo, productivo y a los practicantes que se encuentran formando parte de la planta de lácteos "El Belén", tuvo una gran acogida, puesto que participaron de manera positiva en las medidas correctivas 
tomadas para el mejoramiento de la empresa, asegurando la inocuidad y calidad de los quesos luego de socializadas las prácticas.

Luego de la aplicación de las medidas correctivas en el proceso de producción del queso fresco, la carga microbiana de los productos tuvieron una considerable disminución, pues antes de la aplicación de BPM se encontraron poblaciones microbianas de Escherichia coli 2,78 UFC/g, Enterobacterias 106,67 UFC/g, Staphylococcus aureus se encontró 291,67 UFC/g y luego se redujeron en su totalidad; ajustándose así todos a los parámetros normales establecidos que la Norma INEN 1528:2012 exige y por ende mejorando su inocuidad y calidad.

Mediante el diseño e implementación de buenas prácticas de manufactura en la planta de lácteos "El Belén", se logró el incremento de la calidad de los quesos frescos producidos en este lugar, además permitió incrementar el cumplimiento de los requisitos establecidos por el MSP 2002 a los establecimientos donde se producen y manipulan alimentos, señalando así la eficiencia en la creación del manual, pues en un principio se obtuvo un nivel de cumplimento global de 54,76\% y luego de implantado las herramientas BPM necesarias el resultado aumentó a un $81,75 \%$.

\section{References:}

1. Anderson, P y otros., Microbiología Alimentaria., Madrid-España., Díaz de Santos., (2000). Pp. 17, 19, 55, 60, 83, 85,141, 143.

2. Anderson, K., Whitlock, L. Y Harwood, Y. (2005).Persistence and Differential Survival of Fecal Indicator Bacteria in Subtropical Waters and Sediments. En: Applied and Environmental Microbiology. Vol. 71, N6, p. 3041-3048. DOI: 10.1128/aem.71.6.3041-3048.

3. Albarracín, F. \& Carrascal, A. (2005). Manual de Buenas Prácticas de Manufactura para microempresas lácteas. Pontificia Universidad Javeriana, Colombia.

4. ARCSA. (2015). Resolución ARCSA-DE-067-2015-GGG. Obtenido de Agencia Nacional de Regulación, Control y Vigencia Sanitaria: https://www.controlsanitario.gob.ec/wpcontent/uploads/downloads/2015/12/Resolucion_ARCSA-DE-0672015-GGG.pdf

5. Axelson, F. Sonrin, M. Transia, L. (2004) Technical Handbook. Sweden: Diffchamb AB.

6. Barrios, H. (2006). Evaluación y mejoramiento de la calidad microbiológica de queso fresco a base de leche no pasteurizada, elaborado artesanalmente y comercializado en la Facultad de Medicina Veterinaria y Zootecnia de la Universidad de San Carlos de Guatemala [en línea] Guatemala-Guatemala. UNIVERSIDAD DE SAN CARLOS DE GUATEMALA. [Consulta: 6 de 
Septiembre

de

2017.] Disponible en:

http://biblioteca.usac.edu.gt/tesis/06/06_2422.pdf.

7. Battro, P. (2010). Quesos Artesanales. Cali-Colombia. Albatros, p 54.

8. Besterfield, D. (2009). Control de Calidad., 1a ed., México-México D.F., Pearson Educación., Pp.45-47.

9. Bille, J., Rocourt, J. Swamitrathan, B. Listeria., Erhysipelotrix \& Kurthia, In Murray, P., Baron, EJ., Ptaller, MA., Tenover,. FC. Yolken, RH. (EDS). (2003). Manual of Clinical Microbiology. 7th. ed. Washington DC: American Society for Microbiology Press, p.346-56.

10. Bonet, B., \&Otros (2008). Libro blanco de los lácteos, MadridEspaña., s edt. Pp. 1-53.

11. Busetti, M., Langbehn, C., \& Suárez, V. (2004). Buenas prácticas de manufactura en queso artesanal de oveja. Madrid, España. Edit. Talleres gráficos de la E.E.A. p 15-20.

12. Campos, M. Sabsay, C. y Otros. (2005). Guía de aplicación de las Buenas Prácticas de Manufactura en Bodegas. Argentina.

13. CATÃO R, Ceballos, B., (2001).Listeria monocytogenes., coliformes totais e fecháis e E. coli no leite cru e pasteurizado de una industria de lacticinios, no estado da Paraíba Brazil). Ciênc Tecnol Aliment: 28187.

14. Castro, K. (2011). Tecnología de Alimentos. 1a Edición. st. Bogotá, Colombia. Edit. Ediciones de la U. pp 56, 108, 109, 114.

15. CONEVAL, 2010. Consejo Nacional de Evaluación de la Política de Desarrollo Social. Dimensiones de la seguridad alimentaria: evaluación estratégica de nutrición y abasto. México. DF.

16. Elika, (2013). Ficha Técnica para Salmonella. Fundación Vasca para la Seguridad Agroalimentaria.

17. Equipo Vértice. (2009). Aplicación de normas y condiciones higiénico- sanitarias en restauración. España: Vértice.

18. FAO, SAGARPA, SEDESOL, CONEVAL, INSP. (2013). Panorama de la seguridad alimentaria y nutricional en México 2012. Informe país.

19. FAO, FIDA, PMA. (2013). El estado de la inseguridad alimentaria en el mundo 2013. El crecimiento económico es necesario pero no suficiente para acelerar la reducción del hambre y la malnutrición.

20. Grijalva, J. (2011). La Industria Lechera en el Ecuador un modelo de desarrollo., retos. Universidad Politécnica Salesiana del Ecuador., Quito- Ecuador. Tesis., Pp 66-70.

21. González, M., (2002). Tecnología para la elaboración de Queso Blanco, Amarillo y Yogurt., Panamá- Panamá., s edt. Pp. 34-58. 
22. Gordon, R. \& Lowy, F. (2008). Pathogenesis of Methicillin Resistant Staphylococcus aureus4. Infection Clin Infect Dis; 46: 350 89ISSN:4. 1537-6591.

23. Gómez, E. d. (2009). Higiene en alimentos y bebidas (5ta ed. ed.). México: Trillas.

24. Gunasekaran, S., \& AK, M. (2003). Cheese Rheology and Texture. CRC Press. Nueva York, EE.UU.437 pp.

25. Iurlina, M.O., \& Fritz, R. (2004). "Microbilogical quality of Por Salut Argentine cheese stored at two temperatura treatments". Lebensmittel Wissencraft und Technologie. 37:739-748.

26. Instituto Nacional de Defensa de la Competencia y de Protección Intelectual. (1999). Norma Técnica Peruana NTP-ISO 2859-1. Procedimientos de muestreo para inspección por atributos. Parte I: Planes de muestreo clasificados por nivel de calidad aceptable (NCA) para inspección lote por lote. Lima: INDECOPI

27. INSTITUTO DE ECUATORIANO DE NORMALIZACION. (1979). Código de Práctica para Manipulación de Alimentos, Quito.

28. IICA. (2012). Serie de Agronegocios. En IICA, Buenas Prácticas de Manufactura. Una guía para pequeños y medianos agroempresarios.

29. INSTITUTO ECUATORIANO DE NORMALIZACIÓN. INEN. (2012). Norma General etiquetado, rotulado de Alimentos., Requisitos., NTE- INEN 1334:2012., Quito- Ecuador., INEN., Pp. 24.

30. INSTITUTO ECUATORIANO DE NORMALIZACIÓN INEN: (2012).Norma General para Quesos Frescos no Madurados., Requisitos., NTE- INEN 1528:2012., Quito-Ecuador., INEN., Pp. 2-6.

31. Jonhson, M. \& Law, B. A. (2011). The fundamentals of cheese technology. En: Law B.A. y Tamime A.Y. (Eds). Technology of cheesemaking. Segunda edició. Wiley Blackwell, Reino Unido.

32. Laciar A, Vaca L, DE CENTOBI ON. (1999). Listeria monocytogenes en alimentos de origen animal. Rev Argent Microbiol: 25-30.21.

33. Llanca, R. (2007). Estudio de la calidad del queso de mano. Caracas: UCV

34. López, J. (2001). Informe de buenas prácticas de manufactura. Riobamba, Ecuador: 1a ed. Edit. Continental.

35. MINISTERIO DE SALUD Y PROTECCIÓN SOCIAL. (2011). Evaluación de riesgos de Listeria monocytogenes en queso fresco. Colombia.

36. MINISTERIO DE SALUD PÚBLICA. (2002). Decreto ejecutivo 3253. Buenas Prácticas de Manufactura para alimentos procesados. Ecuador. 
37. Puertas, G. Y Mateos, F. (2011).Enterobacterias., Albacete-España., s edt. Pp. 3426-3431.

38. Quispi, Y. (2015). Diseño E Implementación De Un Manual De Buenas Prácticas De Manufactura (Bpm) Y Procedimientos Operativos Estandarizados De Saneamiento (Poes) En La Planta De Lácteos Letilac. FCP. Escuela Superior Politécnica de Chimborazo.

39. Ramírez, D. (2010). Elaboración de yogurt. 1a ed. st. Lima, Perú. Edit. Macro E.I.R.L. pp 66, 67, 68, 91.

40. Revilla, A. (1982). Tecnología de la Leche. Procesamiento, Manufactura y Análisis. 2da Ed. Costa Rica: LEVANTEX S.A.

41. Roberts, A., Pierson, M.D., Marcy, J.E., Alvarado, C.Z Y Summer S .S. (2002). The effect of sorbic acid $n$ the survival of E. coli O157:H7; Salmonella: L. monocytogenes and S. aureus on shredded cheddar and mozzarella cheese". M.S. Thesis. Virginia Polytechnic Institute and State University.

42. Rosero. (2012). Manual de buenas prácticas de manufactura para una empresa láctea. Ibarra-Ecuador. Universidad Técnica del Norte.

43. Sanz, J. L. (2012). Seguridad e higiene en la manipulación de alimentos. Madrid: Paraninfo S.A.

44. Scoott, R., Robinson, R.K. Y Wilbey, R.A. (2002). "Fabricación de queso". Ed. Acribidia, Zaragoza, España.

45. USDA. (2011). Manual de Buenas Prácticas de Manufactura para la Industria Láctea Artesanal. / Proyecto de Apoyo a la Industria Láctea Artesanal.

46. Watson, D. (1994). Revisiones sobre ciencia y tecnología de los alimentos. Volumen 1: Higiene y Seguridad Alimentaria. Zaragoza: Acribiá.

47. Yépez, O. (2010). REVISTA INFORMATIVA., "Elaboración de Queso Fresco de Leche de Cabra". Cuaderno del productor., CaracasVenezuela., No.1., Pp. 68.

48. Scallan, E y Otros. (2012). Revista Médica. "Foodborne Illness Acquires in the United States Major Patogens". Emerging Infectiud Diseases., Washington - E.U.A., N${ }^{\circ} 17$. Pp. 12 -13.

49. Yuste, J y Fung, D. (2002). "Inactivation of Listeria Monocytogenes Scott A 49594 in Apple juice supplemented woth cinnamon". Journal of Food Protection 65: 1663 - 1666. 